Laser Chem., 1999, Vol. 19, pp. 389-392 Reprints available directly from the publisher Photocopying permitted by license only
(C) 1999 OPA (Overseas Publishers Association) N.V. Published by license under the Harwood Academic Publishers imprint, part of The Gordon and Breach Publishing Group.

Printed in India.

\title{
SPECTRAL HOLE BURNING AND EVIDENCE FOR VIBRATIONAL ENERGY MIGRATION OF H-BONDED MOLECULES OBSERVED WITH TRANSIENT INFRARED SPECTROSCOPY
}

\author{
R. LAENEN* and C. RAUSCHER \\ Physik Department E11, Technische Universität München, \\ James-Franck-Str., 85748 Garching, Germany
}

(Received 4 April 1997)

\begin{abstract}
Via intense ps-excitation of the $\mathrm{OH}$-stretching vibration of ethanol diluted in $\mathrm{CCl}_{4}$ we succeed in spectral hole burning within the inhomogeneously broadened oligomer band. The lifetime of the transient hole and the $v=1$-level of the $\mathrm{OH}$-stetch of associated ethanol molecules are determined to be about 1.2 and $1.7 \mathrm{ps}$, respectively. Further dynamical features are discussed which give evidence for energy migration within the $\mathrm{H}$-bonded ethanol chains and subsequent breaking of the oligomers into shorter fragments.
\end{abstract}

Keywords: Hole burning; hydrogen bonding; transient infrared spectroscopy

Infrared excitation with ultrashort light pulses offers the possibility to study directly with time [1], frequency [2-5] and polarization resolution [6] the spreading of vibrational energy past resonant excitation of a selected molecular subensemble. In this paper experimental data will be presented on ethanol dissolved in $\mathrm{CCl}_{4}$ at a notably lower concentration $(0.17 \mathrm{M})$ compared to previous investigations $[7,8]$. Our experimental system is based on a flashlamp-pumped, Kerr-lens mode-locked $\mathrm{Nd}$ :YLF laser with repetition rate of $50 \mathrm{~Hz}$. Via two optical parametric oscillators and subsequent two-stage optical parametric amplifiers

\footnotetext{
*Corresponding author. e-mail: robert.laenen@physik. tu-muenchen.de
} 
(OPA) with simultaneous conversion further down into the infrared we derive IR pump (probe) pulses of $\approx 2$ ps $(1 \mathrm{ps})$ and energy up to $10 \mu \mathrm{J}$ $(10 \mathrm{~nJ})$ in the range of 1600 to $3700 \mathrm{~cm}^{-1}$. Blocking every second pump pulse we are able to detect the induced, relative transmission change of the sample $\operatorname{In}\left(T / T_{0}\right)$, where $T$ and $T_{0}$, respectively, denote the measured energy transmission of the probe pulse with and without excitation of the sample. All measurements are performed at a low excitation level to reduce heating of the sample via deposition of vibrational energy. Data are taken at room temperature with fixed excitation at $3340 \mathrm{~cm}^{-1}$.

Results on the signal transients measured at different prominent frequency settings of the probe pulse are presented in Figure 1. Probing at the frequency position of the pump (a) the signal increases within the experimental time resolution, followed by a first decay of

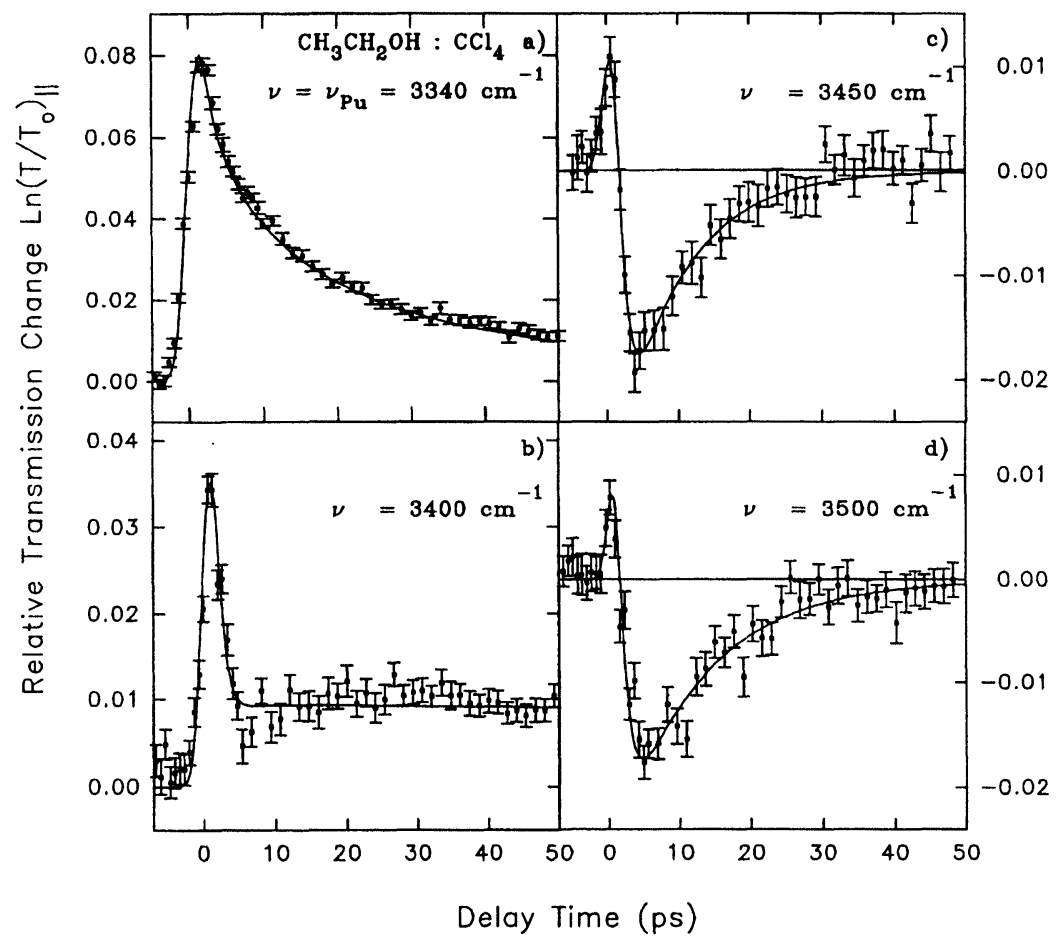

FIGURE 1 The temporal evolution of the signal transients is shown in this figure for four prominent frequency settings of the probe ranging from $\nu=\nu_{\mathrm{Pu}}$ (a) up to $\nu=3500 \mathrm{~cm}^{-1}$ (d) Details see text. 
$\tau_{h}=1.2 \pm 0.2 \mathrm{ps}$ representing the hole lifetime and a subsequent slow decay with $\tau_{i s}=23 \pm 3 \mathrm{ps}$. Structural relaxations proceeds within about $8 \mathrm{ps}$ as inferred from the induced anisotropy of the same data. Data on the dynamics in the blue wing of the spectral hole are presented in Figures $1 \mathrm{~b}-\mathrm{d}$. Probing at $3400 \mathrm{~cm}^{-1}$ (b) a first fast bleaching of the sample close to zero delay time is found, which rapidly decays to a long-lived finite amplitude. A decay time of $\tau_{\text {mig }}<1$ ps is inferred from the data by comparison with model computations. Increasing the probe frequency to $3450 \mathrm{~cm}^{-1}$ (c) the fast bleaching feature of Figure $1 \mathrm{~b}$ has decreased and is now followed by an induced absorption. The latter disappears in turn with an relaxation time of $9 \pm 2$ ps. Further increase of the probing frequency to $3500 \mathrm{~cm}^{-1}$ (d) yields a similar transient: a fast bleaching of the sample around zero delay time, furtherly decreased in peak amplitude, followed by an induced absorption, now vanishing with $14 \pm 2 \mathrm{ps}$.

From the experimental data we develop the following picture [9]: Via excitation of a selected ensemble of molecules we observe transient hole burning in the homogeneously broadened $\mathrm{OH}$-band with a holelifetime of $1.2 \mathrm{ps}$. The spectral hole, which has been also observed in experiments with even higher ethanol concentration of $1.2 \mathrm{M}[8]$ and 2.4 M [10], exhibits a width of about $45 \mathrm{~cm}^{-1}$ and displays the local disorder of the $\mathrm{H}$-bonds due to varying bond angles and distances. The transient hole vanishes mainly via the proposed migration of vibrational quanta along the chain of associated molecules with a time constant $\tau_{\text {mig }}<1 \mathrm{ps}$, as deduced from the bleaching component shown in Figures $1 \mathrm{~b}-\mathrm{d}$ and the fact, that reorientation of the molecules is noteable slower. The energy migration results in an almost isotropic broad bleaching component around $3300 \mathrm{~cm}^{-1}$ which is measured even at early delay times. Subsequent breaking of the ethanol oligomers into shorter chains or rings occurs with a predissociation time of about $1 \mathrm{ps}$ while reassociation in turn proceeds within 9-14 ps depending on the individual structure.

\section{References}

[1] Li, M., Owrutsky, J., Sarisky, M., Culver, J. P., Yodh, A. and Hochstrasser, R. M. (1993). J. Chem. Phys., 98, 5499.

[2] Graener, H., Dohlus, R. and Laubereau, A. (1987). Chem. Phys. Lett., 140, 306. 
[3] Tokmakoff, A., Zimdars, D., Urdahl, R. S., Francis, R. S., Kwok, A. S. and Fayer, M. D. (1995). J. Phys. Chem., 99, 13310.

[4] Arrivo, S. M. and Heilweil, E. J. (1996). J. Phys. Chem., 100, 11975.

[5] Bonn, M., Bakker, H. J., Kleyn, A. W. and van Santen, R. A. (1996). J. Phys. Chem., 100, 15301.

[6] Graener, H., Seifert, G. and Laubereau, A. (1990). Chem. Phys. Lett., 172, 435.

[7] Graener, H., Ye, T. Q. and Laubereau, A. (1989). J. Chem. Phys., 90, 3413.

[8] Laenen, R., Rauscher, C. and Laubereau, A. (1997). J. Phys. Chem., 101A, 3201.

[9] Laenen, R. and Rauscher, C. (1997). J. Chem. Phys., 106, 8374.

[10] Seifert, G., Weidlich, K., Hofmann, M., Zürl, R. and Graener, H. (1996). J. Chim. Phys., 93, 1763. 